\title{
La trastienda de la enseñanza de lenguas extranjeras
}

García Marcos, F. 2019. La trastienda de la enseñanza de lenguas extranjeras. Granada: Comares, 2018, 120 pp.

La trastienda de la enseñanza de lenguas extranjeras constituye parte de una larga lista de producción especializada del autor. Francisco García Marcos es doctor en filología por la Universidad de Granada, con una tesis sobre variación sociolingüística, y catedrático de Lingüística General en la Universidad de Almería. Sus principales preocupaciones han estado enfocadas hacia los campos de la teoría y la metodología lingüística con especial atención a la sociolingüística. Sin embargo, nunca ha dejado de transitar demasiado lejos de la enseñanza de lenguas extranjeras por las experiencias docentes tanto en la Universidad de Kiel, en la entonces República Federal de Alemania, encargándose de la enseñanza de español, como en la Universidad de Granada y, posteriormente, en la Universidad de Almería, donde fundó el Centro de Lenguas y el primer máster de español como lengua extranjera. Este trabajo parte de esa experiencia como docente en lenguas extranjeras, tal y como menciona explícitamente el autor.

Esta obra constituye una aportación novedosa en el campo de la enseñanza de lenguas extranjeras, con aclaraciones críticas de ciertos estereotipos en esa área. Quizá la mayor de ellas sea que se concentra en el docente de la enseñanza de lenguas extranjeras, al que se ha prestado poca 


\section{J. Zou}

Reseña a García Marcos, F. 2019. "La tastienda de la enseñanza de lenguas extranjeras" Sabir. International Bulletin of Applied Linguistics, 1: 190-200

atención antes. Además, nos da una visión panorámica de la enseñanza de lenguas extranjeras abarcando, por un lado, los fundamentos teóricos desde los orígenes hasta final del siglo XX, por otro lado, el itinerario que cumplen los docentes en este ámbito, comprendiendo los fundamentos prácticos, comenzado por el diseño curricular y programación y terminando en los materiales y tareas.

La obra está perfectamente estructurada en cuatro capítulos, la introducción y la bibliografía. García Marcos comienza con un repaso cronológico por sus nutridas experiencias en la enseñanza de lenguas extranjeras que se han mencionado antes, que le llevó a escribir las reflexiones que su propia práctica docente le iba suministrando. A continuación, el autor indica dos constantes que ha mantenido en los trabajos: la primera, la enseñanza de lenguas extranjeras es domino propio y sustantivo de la lingüística aplicada; la segunda, los vínculos directos y tangibles con su propia experiencia como docente. No puedo estar más de acuerdo con este punto de vista. Las declaraciones de principios sobre cómo enseñar una lengua extranjera debe originarse de entre las prácticas de enseñanza de lenguas extranjeras. Al final de la introducción, el autor menciona que esta obra se ha concentrado en los docentes, porque es una dimensión menos atendida de la enseñanza de lenguas extranjeras. Por consiguiente, toda la obra se desenvuelve acerca de este eje.

A continuación, empieza explícitamente lo que es el cuerpo del libro, cuyo título es "El fundamento teórico de la enseñanza de lenguas y sus consecuencias". Presenta los orígenes, el desarrollo y la actualidad de las teorías de la enseñanza de lenguas extranjeras.

Comienza con la aparición de la lingüística aplicada a la enseñanza de lenguas extranjeras a principios de la década de los 60 en Estados Unidos por 


\section{J. Zou}

Reseña a García Marcos, F. 2019. "La tastienda de la enseñanza de lenguas extranjeras" Sabir. International Bulletin of Applied Linguistics, 1: 190-200

"la necesidad que tenían sus tropas desplazadas de aprender los idiomas de los lugares donde recalaban, sobre todo en Europa" (p.6). Cabe mencionar que antes de esta época ya existían actividades docentes de lenguas extranjeras y obras orientadas a este campo, sin embargo, únicamente a partir de los 60 en Estados Unidos "existe una ubicación en la taxonomía disciplinar de la lingüística reservada para la enseñanza de lenguas extranjeras" (p.6).

Luego, presenta el primer paso teórico: el modelo estructuralista. Por más que se le hayan puesto reparos a la metodología estructuralista hoy por sus mayores o menores limitaciones, no se puede negar el logro estructuralista de anclar la enseñanza de lenguas extranjeras en su ubicación taxonómica adecuada, la lingüística aplicada.

A continuación, el autor nos presenta la novedad que supuso en su tiempo la aparición del modelo comunicativo. Con la publicación Teaching Language as Communication de $\mathrm{H}$. Widdowson en 1978 el modelo comunicativo en la enseñanza de lenguas extranjeras se estrenó en la historia. A favor de los planteamientos de conceptos como los de competencia comunicativa y evento comunicativo, formulado por Hymes y Gumperz, el modelo comunicativo tuvo influencias revolucionarias en la enseñanza de lenguas extranjeras, empezando a considerar los vínculos entre una buena comunicación y componentes de la interacción lingüística, como el psicológico, el pragmático, el discursivo y el sociolingüístico. A base de las propuestas de los metodólogos comunicativos, surgieron nuevos materiales, nuevos currículos en los centros, nuevos profesores, nuevos libros. "Por encima de todo ello, el movimiento comunicativo traía consigo un nuevo enfoque de enseñanza, derivado de una manera diferente de entender el lenguaje y las lenguas" (p.9). A pesar de reconocer los grandes logros del modelo comunicativo, el autor muestra la preocupación por la posición académicamente hegemónica que ocupa. A mi 


\section{J. Zou}

Reseña a García Marcos, F. 2019. "La tastienda de la enseñanza de lenguas extranjeras" Sabir. International Bulletin of Applied Linguistics, 1: 190-200

juicio, es acertada esa manera dialéctica de entender el modelo comunicativo. Es verdad que "lo comunicativo aportaba una ostensible solidez teórico y metodológica" (p.9). Sin embargo, debido a que la situación real de la enseñanza de lenguas extranjeras es muy compleja por la heterogeneidad de la sociedad, del profesorado y del alumnado, el modelo comunicativo no es la Biblia que concuerda a todos los posibles casos.

El último apartado de este capítulo se inicia con un caso práctico de integración disciplinar. A continuación, menciona el autor desarrolla la idea de los vínculos que la lingüística y la enseñanza de lenguas mantienen con los otros componentes de la interacción lingüística antes mencionados, la psicolingüística, la pedagogía, la sociología, etc. Desde luego, indica que es menester conocer los mecanismos psicológicos en la adquisición de una lengua y disponer de las herramientas didácticas más modernas. Al final de este capítulo el autor muestra una gran amplitud de miras, ponderando la interdisciplinariedad, sin renunciar a ubicar inequívocamente la enseñanza de lenguas extranjeras dentro la lingüística aplicada.

El segundo capítulo, "La adquisición de lenguas extranjeras como proceso" es otro apartado más bien teórico en que explica el proceso de "cómo se produce en el interior del individuo la incorporación, y el desarrollo, de la competencia comunicativa necesaria para desempeñarse en el uso de una lengua que no es la adquirida en el hogar familiar" (p.13). De la perspectiva procesual se derivan dos consecuencias: el aprendizaje y la adquisición. Estoy a favor del punto de vista del autor en que no es pertinente una contraposición drástica entre estos dos conceptos. El aprendizaje y la adquisición no actúan aislados sino pueden complementarse en el proceso de dominar una lengua extranjera. A pesar de las disputas, "la singladura procesual continuó adelante porque se aprestó a delimitar tanto la secuencia como la caracterización de la 


\section{J. Zou}

Reseña a García Marcos, F. 2019. "La tastienda de la enseñanza de lenguas extranjeras" Sabir. International Bulletin of Applied Linguistics, 1: 190-200

evolución de la adquisición de lenguas extranjeras, gracias fundamentalmente a la incorporación de los conceptos de estilo e interlengua" (p.15).

A continuación, en el primer apartado de este capítulo nos menciona que Taronne planteó el concepto de continuum estilístico en la adquisición de lengua extranjera. Definió los dos estilos: vernáculo y cuidadoso, que el primero, se caracteriza por menos variabilidad y atención a la fórmula lingüística, y el segundo al revés. Conforme a este planteamiento, los estudiantes se iniciarían en el cuidadoso para ir integrándose en el vernáculo. El autor cree que este proceso está contra de la realidad sociolingüística, delimitada justo al contrario. Sin embargo, reconoce lo positivo de la gradualidad de los procesos de adquisición de lenguas extranjeras. Además, el autor incorpora los conceptos de códigos restringidos y códigos elaborados al campo de la adquisición/aprendizaje de lenguas extranjeras, más adecuados para describir este proceso, empezando por los restringidos hasta alcanzar los elaborados. Al mismo tiempo, propone la idea de "acomodarse una delicada y costosa tarea de elaboración del input" (p.18) y "ampliar la noción de situación de comunicación" (p.18) para llegar al objetivo de "adiestrar a los estudiantes en el desarrollo de una competencia estilística aceptable en la lengua objeto" (p.18).

El último apartado de este capítulo trata de la interlengua que es otro punto relacionado la enseñanza de lenguas extranjeras con algunos conceptos sociolingüísticos. La interlengua se produce en el proceso de aprendizaje/adquisición de lenguas extranjeras caracterizando por una mezcla de la lengua materna y la lengua meta. En este sentido, sería similar al pidgin. Sin embargo, los dos no son lo mismo y existen diferencias esenciales. Primero, los usuarios de la "interlengua" serían alumnos de lenguas extranjeras, cuyo objeto consiste en dominar la lengua meta a un alto nivel y disminuir los errores 


\section{J. Zou}

Reseña a García Marcos, F. 2019. "La tastienda de la enseñanza de lenguas extranjeras" Sabir. International Bulletin of Applied Linguistics, 1: 190-200

con el transcurso del tiempo. En cuanto al "pidgin" los usuarios serían la gente común, sin adiestramiento específico en lenguas extranjeras, cuyo objetivo en esta ocasión sería "hacer posible la comunicación entre hablantes de dos lenguas distintas para solventar necesidades comunicativas concretas" (p.22). Segundo, los dos tipos de usuarios tienen distintos estratos en sus contextos sociales, o sea, el primero tiene una relación simétrica de poder lingüístico, pero el otro no. Tercero, la interlengua cambiaría según las necesidades de comunicación del usuario, de lo simple a lo complejo, de lo bajo a lo alto, dejando gradualmente la lengua materna y acercándose a la lengua meta. El desarrollo del pidgin tiene dos resultados diferentes: uno es desaparecer con el cambio del sistema social y el otro es adquirido por descendientes, convirtiéndose en criollo que forma una nueva forma lingüística. En fin, "el núcleo de la actividad pidginizadora y criollizadora es prioritariamente social, con independencias de que sean hablantes concretos quienes lo desarrollen, mientras que la adquisición de una lengua extranjera es una actividad preferentemente individual, con independencia en este caso de que sobre ella concurra, con diferente intensidad, otros condicionamientos sociales" (p.24).

El capítulo tercero titulado "La incorporación del acento didáctico" es la parte nuclear de esta obra, acerca de los componentes imprescindibles en las actividades docentes de lenguas extranjeras. Este capítulo está dividido en cuatro apartados empezando con "Diseño curricular y programación". El primer problema con el que se enfrenta el diseño curricular es decidir el modelo de lengua mediante el que realizar la instrucción y situarlo como objetivo de aprendizaje debido a que existen variedades y dialectos además de la lengua estándar. Naturalmente se toma la lengua estándar como el modelo de lengua, sin embargo, si las aulas de lenguas extranjeras han de incorporar otras variedades, el autor indica que debe prestar atención a que "en qué lugar exacto 


\section{J. Zou}

Reseña a García Marcos, F. 2019. "La tastienda de la enseñanza de lenguas extranjeras" Sabir. International Bulletin of Applied Linguistics, 1: 190-200

del diseño curricular se incluirán esos objetivos, cómo se suministrarán a los estudiantes y, en último término, si completarán o no todas las destrezas" (p.32). Además, el autor explica que no es adecuado enseñar a partir de toda la diversidad dialectal de una lengua meta. Incluso los hablantes maternos no pueden dominar todos los repertorios funcionales de todas las comunidades de habla su propia lengua, y la competencia dialectal se va desarrollando a través de la adquisición lingüística del idioma propio. Por último, el autor aclara un tópico de la enseñanza de lenguas extranjeras: la enseñanza de lenguas extranjeras puede desarrollar una competencia comunicativa completa en la lengua meta. La investigación empírica ha demostrado que hay componentes de la competencia comunicativa que solo se adquieren mediante inmersión prolongada en la lengua meta.

A continuación, nos presenta los componentes del diseño curricular abarcando los contenidos lingüísticos, los funcionales, los culturales y las estrategias comunicativas y pedagógicas que "han de estar engarzados de modo secuencial: permitiendo el tránsito desde lo más elemental, lo más primario comunicativamente, a lo más elaborado y complejo" (p. 35). Una vez que está determinado el diseño curricular, hay que realizarlo mediante la programación teniendo en cuenta los objetivos, los contenidos que se impartirán, las metodologías, las actividades y la evaluación del mismo.

El siguiente apartado de este capítulo trata del tema: el profesorado que es el protagonista encargado de llevar a cabo el diseño curricular. El autor comienza con las posibles tipologías del docente en lenguas extranjeras. Según E. Martín Peris (1990), el profesorado se divide en cuatro tipos, en función de su posición en la dirección de la clase: el profesor autoritario, el autoritativo, el asambleario y el abdicado. F. García Marcos indica que solo las dos opciones centrales están en condiciones de propiciar autonomía de aprendizaje. En estos 


\section{J. Zou}

Reseña a García Marcos, F. 2019. "La tastienda de la enseñanza de lenguas extranjeras" Sabir. International Bulletin of Applied Linguistics, 1: 190-200

dos casos, los alumnos no solo aprenderán contenidos de la lengua meta sino también adquirirán destrezas en ella. Además, el autor enfatiza que existe un necesario componente de tinte humanístico en la enseñanza de lenguas extranjeras, porque las actividades docentes son interpersonales en las que crecen relaciones interpersonales. Luego ejemplifican más responsabilidades del profesorado referidas a la renovación comunicativa, a la conciencia didáctica y a la distancia cultural. Estas experiencias prácticas y exitosas muestran los problemas concretos y las soluciones aconsejables de los que podemos sacar inspiraciones los jóvenes docentes de lenguas extranjeras.

El próximo apartado del tercer capítulo trata del aula, que es un ámbito físico y psicológico en que se desarrollan las actividades didácticas. Durante las últimas décadas, los docentes de lenguas extranjeras empezaron a distinguir escenario y escena, consideración que los llevó a entender el aula como un evento comunicativo. A través de la incorporación de las nuevas tecnologías como vídeos, audios o TIC's en la enseñanza de lenguas extranjeras, las distancias entre el aula y el desempeño idiomático cotidiano están cada día más reducidas. Sin embargo, el autor indica que "el problema subyacente radica en que las soluciones metodológicas que puedan aportarse no dejan de constituir aproximaciones ficcionales de la realidad idiomática" (p.42). Refiere F. García Marcos la metodología alternativa que propuso en 1989, en la que trataba de transformar el tradicional concepto del aula al introducir hablantes ajenos a ella. El objetivo consistía en aportar al estudiante extranjero diálogos reales más cercanos a la realidad idiomática de la lengua meta. Para comprobar esta hipótesis, realizó una investigación empírica de comparación los resultados de la aplicación de la metodología convencional y la propuesta. Los resultados del experimento mostraron que las mejoras metodologías "palían pero no zanjan, el déficit entre las destrezas lingüísticas de los estudiantes extranjeros dentro y 


\section{J. Zou}

Reseña a García Marcos, F. 2019. "La tastienda de la enseñanza de lenguas extranjeras" Sabir. International Bulletin of Applied Linguistics, 1: 190-200

fuera del aula" (p.45). Al final, el autor propone que "las teorías pedagógicas deben forzosamente confortarse con la práctica de aula" (p.46) debido a la diversidad del alumnado.

La última parte de este capítulo trata del otro protagonista del proceso educativo: el alumnado. Primero, menciona que todo currículo, toda enseñanza de lenguas extranjeras está orientada hacia el alumno. Segundo, el autor habla de la negociación que ha sido un lugar común de la bibliografía especializada desde hace décadas. El autor nos presenta los dos puntos de vista de la negociación que no deja de ser un tema polémico. Para comprobar la eficacia de la negociación, el autor hizo dos experimentos para descender a la realidad empírica, medir, contar y valorar. El primero trata de la aplicación de la negociación a tres grupos de estudiantes alemanes y el segundo a tres grupos distintos de estudiantes universitarios en situación de inmersión en España. De estos experimentos sacó los resultados siguientes. En general, la negociación no fue tan eficaz como se esperaba en principio. Los adolescentes se sintieron más motivados por las prácticas comunicativas y se beneficiaron de ellas. Sin embargo, los universitarios filólogos neutralizaron la metodología. Además, la heterogeneidad cultural del grupo potencia el desarrollo de actividades comunicativas. Por último, los estudiantes en situación de no inmersión prefieren tendencialmente la dramatización o la negociación. A base de esos resultados, el autor llama a los profesores de lenguas extranjeras a "conocer al grupo, dialogar con sus integrantes, tratar de aproximarse a sus inquietudes y condicionamientos, antes de entrar en los detalles, que pueden variar de un grupo a otro, de un tiempo a otro" (p.53).

El siguiente apartado de este capítulo se titula "El nuevo estatus del error en el marco de renovación pedagógica". En todo proceso humano, incluida la ciencia, es imposible evitar errores. Incluso estos pueden convertirse en factores 


\section{J. Zou}

Reseña a García Marcos, F. 2019. "La tastienda de la enseñanza de lenguas extranjeras" Sabir. International Bulletin of Applied Linguistics, 1: 190-200

útiles mediante la reflexión sobre las causas y cómo corregirlos. La nueva imagen del error en el aula de lenguas extranjeras pide que se clausure la tradicional mentalidad punitiva y se evite cometer errores mediante actividades diversas. El autor muestra su preocupación sobre esa situación. A su juicio, hay que quedarse en el medio entre el énfasis intenso en la docencia y el formalismo de algunos esquemas lingüísticos.

El último apartado del capítulo trata de la heterogeneidad del alumnado de lenguas extranjeras que es otro punto del que debe tomar consciencia. Como menciona el autor, frecuentemente se comete la identificación casi exclusiva de enseñanzas de lenguas extranjeras con niveles superiores. En este caso, se ignoran las distintas necesidades y motivos de los alumnos: curiosidad, deseos de viaje al país de la lengua meta, negociación, etc. Además, el autor nos presenta la posible taxonomía de los alumnos. Prestar atención a la heterogeneidad del alumnado nos ayuda a los docentes a ajustar la enseñanza de lenguas extranjeras según las necesidades de los alumnos.

El capítulo cuarto titulado "Materiales y tareas" es la última parte de esta obra. En este capítulo el autor establece los fundamentos empíricos de los materiales. Con datos descriptivos y experimentos empíricos el autor nos justifica la necesidad de prestar atención a la frecuencia del uso en la cotidianidad idiomático para fijar los objetivos concretos y los contenidos de los materiales de la enseñanza de lenguas extranjeras. Además, nos presenta el enfoque por tareas, sus ventajas y "la delicada cuestión de elaborar tareas eficientes, atractivas y pertinentes" (p.64) y nos muestra un ejemplar: gramática novelada que diseñó y aplicó en la Universidad de Granada. Este capítulo termina con la presentación de los nuevos canales de la enseñanza de lenguas extranjeras: la informatización y la realidad virtual inmersiva.

Como se ha indicado al principio de la reseña, esta obra destaca, en primer 


\section{J. Zou}

Reseña a García Marcos, F. 2019. "La tastienda de la enseñanza de lenguas extranjeras" Sabir. International Bulletin of Applied Linguistics, 1: 190-200

lugar, por la temática que presenta, que ofrece una visión panorámica de la enseñanza de lenguas extranjeras. En segundo lugar, el autor aclara unos estereotipos teóricos, metodológicos y prácticos mediante convincentes resultados de experimentos empíricos. Finalmente, esta obra nos provee una novedosa perspectiva combinada en relación la enseñanza de lenguas extranjeras, debido a las abundantes experiencias como investigador del aula, docente de lenguas extranjeras y como sociolingüista. El libro, en conclusión, es una obra maravillosa, que es sumamente recomendable para los docentes de lenguas extranjeras y para cualquier investigador interesado en este campo de estudio. 